\title{
Fractional anisotropy distributions in 2- to 6-year-old children with autism
}

\author{
C. Cascio ${ }^{1,}$, , M. Gribbin ${ }^{2,}$, , S. Gouttard ${ }^{3}$, R. G. Smith ${ }^{4}$, M. Jomier ${ }^{5}$, S. Field ${ }^{6}$, M. Graves ${ }^{4}$, H. \\ C. Hazlett ${ }^{4}$, K. Muller ${ }^{7}$, G. Gerig ${ }^{3}$, and J. Piven ${ }^{4}$ \\ ${ }^{1}$ Psychiatry and Kennedy Center for Research on Human Development, Vanderbilt University, \\ Nashville, Tennessee, USA \\ ${ }^{2}$ Department of Biostatistics, Human Genome Sciences, Rockville, Maryland, USA \\ ${ }^{3}$ Department of Computer Science, University of Utah, Salt Lake City, Utah, USA \\ ${ }^{4}$ Psychiatry/Carolina Institute for Developmental Disabilities, University of North Carolina, Chapel \\ Hill, North Carolina, USA \\ ${ }^{5}$ Department of Computer Science, University of North Carolina, Chapel Hill, North Carolina, USA \\ ${ }^{6}$ Frank Porter Graham Child Development Institute, Chapel Hill, North Carolina, USA \\ ${ }^{7}$ Department of Epidemiology and Health Policy Research, University of Florida, Gainesville, \\ Florida, USA
}

\section{Abstract}

Background-Increasing evidence suggests that autism is a disorder of distributed neural networks that may exhibit abnormal developmental trajectories. Characterisation of white matter early in the developmental course of the disorder is critical to understanding these aberrant trajectories.

Methods-A cross-sectional study of 2- to 6-year-old children with autism was conducted using diffusion tensor imaging combined with a novel statistical approach employing fractional anisotropy distributions. Fifty-eight children aged 18-79 months were imaged: 33 were diagnosed with autism, 8 with general developmental delay, and 17 were typically developing. Fractional anisotropy values within global white matter, cortical lobes and the cerebellum were measured and transformed to random $F$ distributions for each subject. Each distribution of values for a region

\footnotetext{
(C) 2012 The Authors.

Correspondence: Dr Joseph Piven, Psychiatry/Carolina Institute for Developmental Disabilities, University of North Carolina, Chapel Hill, NC 27599, USA (joe_piven@med.unc.edu).

These authors made equal contributions to this work.

Financial disclosures

None of the authors have conflicts of interest to declare.

Supporting information

Additional Supporting Information may be found in the online version of this article:

Details on theory and implementation of statistical analysis.

Figure S1 A: Plots illustrating the fits of the Beta distributions to the transformed global FA distributions for six randomly chosen subjects. B: The $F$-distribution fit to transformed global FA values for the same six subjects.

Table S1 Tests of whole brain white matter (global) main effects model, with $\delta$ as the outcome variable

Table S2 Tests of lobes main effects model, with $\delta$ as the outcome variable

Table S3 Group comparisons of 8 by lobe

Table S4 Tests of cerebellum main effects model, with $\delta$ as the outcome variable

Please note: Wiley-Blackwell are not responsible for the content or functionality of any supporting materials supplied by the authors.

Any queries (other than missing material) should be directed to the corresponding author for the article.
} 
was summarised by estimating $\delta$, the estimated mean and standard deviation of the approximating $F$ for each distribution.

Results-The estimated $\delta$ parameter, $\delta$, was significantly decreased in individuals with autism compared to the combined control group. This was true in all cortical lobes, as well as in the cerebellum, but differences were most robust in the temporal lobe. Predicted developmental trajectories of $\delta$ across the age range in the sample showed patterns that partially distinguished the groups. Exploratory analyses suggested that the variability, rather than the central tendency, component of $\delta_{\mathrm{w}}$ as the driving force behind these results.

Conclusions-While preliminary, our results suggest white matter in young children with autism may be abnormally homogeneous, which may reflect poorly organised or differentiated pathways, particularly in the temporal lobe, which is important for social and emotional cognition.

\section{Keywords}

autism; brain; developmental; diffusion tensor imaging; fractional anisotropy; white matter

\section{Introduction}

Autism is a developmental disorder that is characterised by an onset in early childhood and a triad of impairments: (1) deficits in reciprocal social interaction; (2) delayed and/or abnormal language and communication skills; and (3) a narrow range of interests, dependence on routine or ritual, and/or repetitive movements (American Psychiatric Association 2000). A complex and heterogeneous disorder, its neural substrates are a subject of ongoing investigation; however, relatively few studies addressing brain structure and function have been performed in young children. The most consistent neurobiological finding is an enlargement of brain volume (Piven et al. 1996; Courchesne et al. 2001; Hazlett et al. 2005), which is detectable as early as 2 years of age, affects both white and grey matter volume, and may be related to decreased axonal pruning early in development (Frith 2003).

Brain imaging studies have also specifically implicated circumscribed networks in relation to certain symptomatic domains, such as temporal (Aylward et al. 1999; Adolphs et al. 2001) and fronto-striatal (Sears et al. 1999; Carper \& Courchesne 2005) networks that may underlie social/emotional and ritualistic/repetitive behaviour, respectively. Recently, functional connectivity studies have addressed the ability of the nodes of such networks to work in concert, and many have suggested that communication between these nodes may be suboptimal in autism (Noonan et al. 2009; Minshew \& Keller 2010).

Increased white matter volume and the implication of neural network integrity raised by functional connectivity studies suggest that there may be differences in white matter tracts forming physical connections between brain areas in autism. Diffusion tensor imaging (DTI) indirectly measures the integrity of these tracts via known tissue water diffusion properties within axons. DTI employs a modification in the application of the standard radio frequency gradients in magnetic resonance imaging (MRI), rendering it sensitive to tissue water diffusion (Basser \& Pierpaoli 1996). In white matter, the myelin sheath and the physical structure of axons impose physical boundaries to the direction of water diffusion, so that diffusion primarily occurs along the axis of the fibre bundles (Beaulieu 2002), rather than in a freely diffusing state. This directionality is represented by a ratio that describes the diffusion along this primary direction relative to the other two orthogonal directions in threedimensional space; the most commonly used variant of this ratio is fractional anisotropy (FA). At each voxel of the image, FA expresses the deviation of the diffusion from that of spherical or isotropic diffusion, characteristic of what would be seen in freely diffusing 
tissue, such as cerebrospinal fluid within the ventricles. Although the relation between FA and underlying histological properties is not fully understood, particularly in areas of crossing or diverging fibres, higher FA measured in the centre of a fibre bundle (where variability is lowest) is generally interpreted to indicate stronger, more coherently bundled, or more heavily myelinated white matter tracts (Beaulieu 2002). By unveiling the integrity and coherence of brain white matter representing fibre projections, DTI has the potential to contribute a much richer understanding of white matter structure than conventional MRI alone.

Studies of white matter development in childhood and adolescence as measured by DTI consistently demonstrate that FA increases with age throughout brain white matter (see review by Cascio et al. 2007), presumably reflecting ongoing myelination during early childhood and increased organisation and coherence of white matter tracts throughout development. DTI may be a particularly promising tool for the study of autism, whose neural underpinnings are likely to involve a distributed network of developing brain systems (Müller 2007). Voxel-based morphometry studies have demonstrated widespread white matter volumetric abnormalities in autism (McAlonan et al. 2005; Mengotti et al. 2011), particularly in limbic regions, supporting the hypothesis that aberrant neural connectivity is a key factor in autism (Courchesne \& Pierce 2005; Minshew \& Williams 2007).

Tractography studies that utilise FA to trace probable fibre paths suggest that fronto-striatal and fronto-limbic circuits (Kumar et al. 2010; Langen et al. 2011) as well as commissural connections (Pugliese et al. 2009; Kumar et al. 2010; Lo et al. 2011) are compromised in autism.

In a preliminary DTI study of high functioning older children and adolescents with autism compared to matched controls (Barnea-Goraly et al. 2004), widespread reduction in mean FA, including regions implicated in autism such as the temporal lobe, was seen throughout the cerebral cortex using a voxel-based analysis method. More recently, Alexander et al. (2007) demonstrated reduced FA in the corpora callosa of a subgroup of individuals with autism, for whom cognitive abilities were lower than both controls and other autism groups. Keller et al. (2007) noted decreased FA in both the corpus callosum and internal capsule of a large sample of adolescents and adults with autism, and Lee and colleagues observed decreased FA in the superior temporal gyrus and temporal stem (Lee et al. 2007). Among other diffusion anomalies, widespread decreases in FA were recently reported in the corpus callosum, internal capsule, anterior thalamic radiation, and inferior and superior longitudinal fasciculi (SLF) in a large sample of children and adolescents with autism spectrum disorder (ASD) (Shukla et al. 2011). Jou and colleagues replicated these findings in another recent study, demonstrating reduced FA in the SLF as well as several other long-range fibre tracts ( Jou et al. 2011). In contrast to these studies which focused on older children, adolescents or adults, a recent study by Ben Bashat et al. (2007) found increased FA in a small $(n=7)$ group of preschoolers with autism compared to typically developing (TYP) controls, suggesting a complex developmental trajectory of white matter pathways in autism. Although this study was preliminary, a recent follow-up study replicated this result (Weinstein et al. 2011). A comprehensive examination of FA in a large sample of young children with autism is important to clarify the picture of early emerging brain differences that may give rise to the disorder.

In the current study, we present DTI data from a large sample of young children with autism compared to a group including TYP children and children with developmental delay (DD) without autism. This sample overlaps with that of a previous study (Hazlett et al. 2005), in which we reported generalised brain volume differences that were particularly robust in white matter. In this report, we also introduce an alternative analysis approach that greatly 
improves statistical power within a neuroimaging framework and provides a means to summarise the entire distribution of FA values within individual regions of interest (ROIs).

The methods used in this study are based on the principle that, because FA values are bounded by 0 and 1 and are calculated from the covariance of a Gaussian distribution that describes water diffusion, FA itself cannot be Gaussian. Thus, using mean FA directly in a parametric mixed model violates its statistical assumptions, and a viable alternative is to describe FA in terms of its distribution among the voxels in a ROI. The rationale for the use of this method is based on first principles of statistics and is described briefly in the Statistical Analysis section. Further detail on the theory and use of this method can be found in Supporting Information and in Clement et al. (2008; Clement-Spychala et al. 2010).

\section{Methods}

\section{Sample}

A subset of the sample used in our longitudinal study of brain development in autism (Hazlett et al. 2005) was used for this study. In this subset, 33 were diagnosed with autism, 8 with DD without autism, and 17 were TYP. During the course of the longitudinal study, an upgraded sequence was adopted; the subset included in this report comprises 66 observations from 58 children who were scanned using the newer DTI sequence. A small number in each group had data from two observation points (three each in the autism and TYP groups, two in the DD group); these observations were separated by 2 years' time. We used a hierarchical model to account for the lack of independence between these repeated observations in eight participants. The participants were from the south-eastern USA and were recruited and characterised as described previously (Hazlett et al. 2005): children with DD were included to enrich the control sample with subjects who were comparable to the subjects with autism in cognitive development.

Subjects with autism were referred after receiving a clinical diagnosis of an ASD; diagnosis was confirmed with the Autism Diagnostic Interview - Revised (ADI-R; LeCouteur et al. 2003) and Autism Diagnostic Observation Schedule (ADOS; Lord et al. 1999) and finalised with an expert clinical DSM-IV diagnosis. All subjects in the autism group met criteria on both the ADOS and ADI-R, as well as received an expert DSM-IV diagnosis of autistic disorder. Subjects with DD were referred only if they had no known identifiable cause for their delay (e.g. prematurity, genetic disorder or neurological disorder) and had no indication of a pervasive developmental disorder. Subjects were excluded for having evidence of a medical condition thought to be associated with autism, including fragile $\mathrm{X}$ syndrome, tuberous sclerosis, gross central nervous system injury (e.g. cerebral palsy, significant perinatal or postnatal complications or trauma, drug exposure), seizures, and significant motor or sensory impairments. Descriptive statistics can be found in Table 1.

\section{Image acquisition and processing}

All scans were acquired on a 1.5 T GE Signa Advantage MR scanner. T1-weighted structural images were acquired using a 3D IR prepped SPGR protocol with a $256 \times 256 \times$ 124 image matrix at $0.78125 \times 0.78125 \times 1.5 \mathrm{~mm}$ resolution. DTI images were acquired using four repetitions of a 12-direction spin-echo single-shot echo planar imaging sequence with a $128 \times 128 \times 30$ image matrix at $1.875 \times 1.875 \times 3.8 \mathrm{~mm}$ resolution (with a $0.4 \mathrm{~mm}$ gap) using a b-value of $1000 \mathrm{~s} / \mathrm{mm}^{2}$.

Each DTI slice was screened for motion and other artefacts using custom software that automatically removed slices or shots falling outside predetermined parameters (http:// www.ia.unc.edu/dev/download/dtichecker/index.htm), including slice-to-slice and averageto-average motion, and to correct for the latter. Subsequently, both the correction of eddy 
current-based image distortions using mutual information-based unwarping and the calculation of the diffusion tensor elements, eigenvectors and eigenvalues were performed using another custom program (Tensorcalc, Stanford University: http://rsl.stanford.edu/ moseley/tensorcalc/). These eigenvectors and values were then used to compute FA maps using a customised program: (http://www.ia.unc.edu/dev/download/fibertracking/ index.htm).

The 66 FA maps were then coregistered into the same coordinate system using both affine (scaling and translation) and non-linear (fluid warping) coregistration. These coregistered FA maps were then combined with a ROI mask comprising a white matter segmentation map and lobe parcellation map created using a semi-automated method (Yushkevich et al. 2006) from a population atlas of all subjects' structural MRI with associated tissue segmentation (Hazlett et al. 2005). The goal of this combination of masks was the isolation of white matter within each of the cortical lobes, and the cerebellum. Because of the lack of clear landmarks to mark the boundary between parietal and occipital lobe, this method yields a parcellation that does not separate these two lobes. This application of white matter mask and lobe parcellation to the individual coregistered FA maps allowed the extraction of FA values for global (whole brain) white matter, white matter in each cortical lobe, and the cerebellum (see Fig. 1).

\section{Outcome variable derivation and statistical analysis}

Raw FA values were transformed to $B$ values, and the distribution of these values was used to approximate a beta distribution using two summary parameters estimated from the distribution of the raw $B$ values (resulting in $B$ values $=B$ values $=\sqrt{I-F A^{2}}$ ); for details, see Supporting Information). The raw FA distributions, transformed to the $B$ variable, are overlaid with the approximated beta distributions in Fig. S1 for six randomly chosen participants. The plots in Fig. S1 illustrate how accurately the transformation represents the raw data, but the bounded nature of the beta distribution resulted in limitations to fit at the boundaries of the distribution (Fig. S1A, top-right and bottom-left). Because of this limitation, beta distributions were then transformed to a non-bounded $F$ distribution using first principles and a well-known statistical transformation meant to spread the data for statistical analysis. An estimated mean and standard deviation of the approximating $F$ were calculated for each distribution. This transformation yields a non-bounded distribution, and unlike the beta value, the $F$ value varies in the same direction as FA, which simplifies understanding of the relationship between the outcome variable and the physical property that it represents. A further strength of this conversion is that summary statistics are used to calculate the $F$ distribution values rather than individual FA values, resulting in an inherently more reliable and stable variable that is both statistically and scientifically sufficient, as evidenced by how well the distributions fit the real data (Fig. S1B).

We used the single value $\delta$, representing the mean plus one standard deviation $(\delta=\mu+\sigma)$ of the $F$ distribution as our outcome variable. $\delta$ and similar robust measures have been used in previous imaging studies (Puff et al. 1994; Clement et al. 2008; Clement-Spychala et al. 2010). Because each statistic is based on thousands of FA data values, the beta and equivalent $F$ moment estimates can be expected to follow Gaussian distributions rather well (a standard consequence of large-sample theory for maximum likelihood estimates; this assumption is confirmed in Fig. S1). General linear mixed models assuming Gaussian distributions of residuals were fitted using the mixed procedure (Brown \& Prescott 2006) in sas (version 8.2) separately for the global white matter, lobe and cerebellum ROIs, based on Restricted Maximum Likelihood Estimation. Group, age and gender served as betweenparticipant predictors for the analysis of all ROIs. For the lobe and cerebellum models, side (hemisphere) was also included as a within-participant predictor, as was lobe in the lobe 
model. The age and gender variables were centred about 4 years and 0.8 respectively, the approximate overall mean age and proportion of male participants.

Our primary planned analysis consisted of three separate models with $\delta$ as the outcome variable for global white matter, individual cerebral lobes and the cerebellum. Further planned analyses included tests of group by lobe interactions, regardless of the outcome of the main lobes model. In secondary analyses, we explored the contributions of slice thickness and cognitive development scores. Finally, a secondary analysis that explored the relative contributions of the components of $\delta(\mu$ and $\sigma)$ was performed.

\section{Results \\ Primary analysis}

Model diagnostic analysis-All assumptions, especially the assumption of Gaussian distribution, must always be checked in a mixed model. Hence for each model we conducted thorough regression diagnostic analysis following the principles outlined in Chapter 7 , Assumption Diagnostics, in Muller \& Fetterman (2002). All results supported the validity of the assumption.

Results for global white matter-For global white matter, the model revealed a statistically significant effect of group $\left(F_{2,54}=7.77, P=0.0011\right)$, with the autism group having a significantly lower $\delta$ than the combined control group $\left(F_{1,54}=14.73, P<0.0003\right)$. This difference seems to be driven by a significant difference between the autism and TYP groups $\left(F_{1,54}=14.65, P<0.0003\right)$. As expected, a significant effect of age $\left(F_{1,54}=39.69, P\right.$ $<0.0001$ ) was also observed. In Fig. 2, predicted change with age in each of the three groups is illustrated. The typical group shows the steepest linear increase in predicted $\delta^{6}$ with age, while the DD group's slope is shallowest; however, there was no significant interaction between age and group. The model output is summarised in Table S1.

Results for cerebral lobes-We analysed voxels within the white matter mask first for all cerebral lobes together in the linear mixed model, and then separately for each lobe in follow-up tests. The linear mixed model (summarised in Table S2) for the lobe analysis revealed that $\delta$ differed significantly by lobe $\left(F_{2,110}=18.23, P<0.001\right)$, group $\left(F_{2,54}=5.21\right.$, $P=0.0085)$, side (hemisphere) $\left(F_{1,320}=145.02, P<0.0001\right)$ and age $\left(F_{1,320}=21.74, P<\right.$ $0.0001)$, while a test for interaction between age and group was nearly significant $\left(F_{2,320}=\right.$ $2.96, P=0.053)$. Follow-up analyses revealed significant differences between the autism and combined control groups $\left(F_{1,320}=8.82, P=0.0032\right)$, reflected in significant differences between both the autism and TYP groups $\left(F_{1,320}=6.26, P=0.0128\right)$, and between the autism and DD groups $\left(F_{1,320}=4.83, P=0.0287\right)$; the overall lower predicted values of $\delta^{8} \mathrm{~m}$ the autism group compared to both control groups are depicted in Fig. 3. A statistically significant interaction between group and age for the DD versus typical groups $\left(F_{1,320}=\right.$ $5.65, P=0.0181$ ) was also observed, but other group by age interactions did not reach significance.

Although there was no overall statistically significant interaction between group and lobe, several significant relationships emerged when the groups were compared with pre-planned $t$-tests, based on previous studies indicating reduced structural integrity of white matter in ASD (Barnea-Goraly et al. 2004, 2010; Keller et al. 2007). The autism and combined control groups differed significantly in the $\delta$ value for all three lobes, as summarised in Table S3. However, when a Bonferroni correction was used to correct for multiple tests, only the group differences in the temporal lobe survived. 
Results for cerebellum-The linear mixed model for the cerebellum analysis (also limited to voxels within the white matter mask) yielded significant effects of group $\left(F_{2,54}=\right.$ $15.57, P<0.0001)$, side $\left(F_{1,54}=63.74, P<0.0001\right)$ and age $\left(F_{1,54}=28.16, P<0.0001\right)$ in $\delta^{d}$ The autism group differed significantly from the combined control group $\left(F_{1,54}=6.00, P=\right.$ $0.0176)$. When analysing the autism group compared to the control groups separately, the autism group was significantly different only from the TYP group $\left(F_{1,54}=4.6, P=0.0364\right)$. The results of the model are summarised in Table S4. No significant interactions between group and age were found; the predicted $\delta$ values as a function of age are illustrated in Fig. 4. As in the previous models, the autism and typical groups follow similar trajectories, but the autism group has a much lower level of predicted $\delta$, while the DD group has a static level of predicted $\delta$ throughout the 5-year span.

\section{Secondary exploratory analyses}

Alternative outcome measures-A separate multivariate model was run using each of the two components of $\delta(F$-mean and $F$-standard deviation) as outcome variables separately, revealing that group differences were driven almost entirely by the standard deviation component. Follow-up group tests for the standard deviation model yielded significant differences for the autism versus TYP $(t=-4.07, P=0.002)$ and autism versus DD $(t=$ $-3.18, P=0.0018)$ groups, but no significant differences for the TYP versus DD group, using $F$-standard deviation $(F-\mathrm{SD})$ as the outcome variable. No significant differences in any group comparisons were found using the $F$-mean as the outcome variable. The $F$-SD outcome variable is a reflection of the variability of FA values within the distribution. The exploratory results suggest that the autism group has similar overall FA levels within a ROI, but less variation in FA compared to the other two groups.

Importantly, we also explored the nontransformed FA distribution properties as outcome measures, which resulted in a substantial loss of power, similar to the loss of power that might be observed in simply taking the mean FA value. The transformation to an $F$ distribution is based completely on the first principles of statistics and allows for the summarisation of the entire data set, which provides greater power to detect specific differences.

Additional predictors-Because the groups' mean estimated IQs differ, we ran the global model with cognitive development score included as a predictor. No significant main effects or interactions between cognitive development score and group were found to predict $\delta$. This provides validation for the consideration of the two control groups as a combined group, although results are also presented for the groups separately.

Although a nominal value of $4 \mathrm{~mm}$ was specified for the $\mathrm{z}$-direction of the diffusionweighted images, we found upon inspection of the data that thickness actually varied between 4.09 and $4.21 \mathrm{~mm}$. This variability was wider than expected, and the source of it was unknown. As a result, each model was also run with slice thickness included as a covariate. Thickness did have a significant effect on the outcome measure $\delta\left(F_{1,54}=9.77, P\right.$ $=0.0029$ ), but the significant differences reported in the above section, as well as the predicted trajectories with age, were not altered by the addition of this covariate. Thus, we are confident that the variability in slice thickness does not represent a confound that affects the group differences highlighted in this report but wish to point out that potential variability in slice thickness is a factor that future experiments should take into account.

\section{Discussion}

This study employed a novel approach for the analysis of diffusion data, focusing on FA distributions rather than mean FA values in the brain ROIs. Using this technique, we noted 
significant group differences in cerebral and cerebellar white matter. The autism group had significantly lower $\delta$ values than the combined control group, both globally, and within specific cortical lobes, as well as in the cerebellum, reflecting lower/more variable anisotropy and thus potentially less efficient white matter transduction, under the hypothesis that the structural property of FA is linked to the functional efficacy of fibre pathways. Although this was not a longitudinal study, the predicted trajectories of $\delta$ between ages 1.5 and 6.5 years followed group-specific patterns, with the DD group exhibiting a flat trajectory and the autism group increasing in a manner more similar to the typical group, despite its lower overall $\delta$ values. The only statistically significant difference in group trajectory was between the typical and DD groups.

The statistical approach we employed is an alternative that addresses the high dimension, small sample size problem inherent in neuroimaging, reducing thousands of voxels accurately to two summary parameters via transformation of the FA values into standard distributions with well-known statistical properties. This analysis method provides considerable sensitivity to detect group differences (Clement-Spychala et al. 2010). In the analysis, we considered $F$ distribution means, the SDs and the combination of these values $(\delta=\mu+\sigma)$ as potential outcome measures. While our primary analysis of $\delta$ revealed significant group differences, closer inspection revealed that these differences were driven almost entirely by the standard deviation component of $\delta$. Therefore, the standard deviation $(F-\mathrm{SD})$ was considered as the main outcome variable for secondary analyses. The exploratory results suggest that the variability of FA values within the distributions was the key factor in distinguishing the groups. This diminished variability in FA may reflect a decrease in the differentiation that typically occurs during brain maturation. Although it is possible that this occurs prenatally as diminished neural proliferation, migration, differentiation of neural progenitor cells into specific types of neurons, or axon guidance to specific targets, it is more likely to occur postnatally, during the period in which additional axon growth is followed by pruning of superfluous connections. It is during the early postnatal period that behavioural differences begin to emerge (DSM-IV) and previous studies implicate early postnatal brain overgrowth (Hazlett et al. 2005), which would be consistent with this idea. Our results are consistent with other DTI studies employing more traditional analysis approaches and finding abnormal developmental trajectories of white matter maturation (Ben Bashat et al. 2007; Shukla et al. 2011). The transformations we applied to the FA values do not change the biological significance of the outcome variable; rather they allow us to describe FA more comprehensively within a circumscribed region of the brain than would be possible with only a mean value, particularly for a non-normal, bounded distribution such as FA.

The data in Table S2 and Fig. 3 suggest that there are different patterns over time that partially distinguish the groups. A nearly significant interaction between age and group was observed, with a significant contrast in the TYP versus DD comparison. Although the small size of the DD group prevents firm conclusions, the impression from these tests and Fig. 3 is that the trajectory is steepest in the TYP group, and shallowest in the DD group, while that of the autism group is intermediate between them.

The lobe analysis revealed that group differences in frontal, temporal and occipital-parietal lobes distinguished the autism group from the combined control group. Both the temporal and frontal lobes have been heavily implicated in autism: the temporal lobe being the hub of a wide neural network mediating social and emotional processes (Bauman \& Kemper 1985; Schultz et al. 2000; Amaral \& Corbett 2003; Boddaert et al. 2004; Mosconi et al. 2005), and the frontal lobe a key structure in executive abilities such as set shifting and cognitive flexibility (Turner 1999; Schmitz et 1. 2006; Shafritz et al. 2008). Although multiple interpretations of reduced FA variability are plausible, one possibility is that it reflects early 
disruption of widespread cortical networks in autism, which is consistent with a growing literature emphasising autism as a cortical disconnection syndrome (Geschwind \& Levitt 2007; Minshew \& Williams 2007). Occipital and parietal cortex, as well as superior temporal sulcus, a site of group differences in Lee et al.'s (2007) study, are largely devoted to processing sensory information, and the evidence of their disruption early in autism suggests that sensory processing deficits should be more closely examined in autism. However, after correcting for multiple comparisons, the only group differences that remained were in the temporal lobe, consistent with many other studies demonstrating that social cognition mediated by the temporal lobe is among the most fundamental deficits in ASD. In spite of this, the direction of difference was quite consistent across all three lobes (see Table S3), suggesting more widespread differences in FA variability that may be detectable with increased power.

We also noted group differences in the cerebellum, with the autism group again displaying significantly lower $\delta$ than both control groups. A similar pattern was also observed when predicting $\delta$ bver time; the autism group did increase predicted $\delta$ with age, but overall $\delta$ was greatly decreased relative to both control groups. Reported volumetric differences of the cerebellum in autism are proportional to overall differences in brain volume (Sparks et al. 2002; Palmen et al. 2005). While cerebellar white matter increase has been reported in young children with autism (Courchesne et al. 2001), we did not observe this in a recent study of a larger sample of 2-year-olds (Hazlett et al. 2005). In spite of this, we find significant differences in the FA distribution in the cerebellum in these same young children, suggesting that the early stages of cerebellar white matter organisation into fibre pathways are somehow altered in autism, without affecting overall white matter volume in the cerebellum. This is consistent with a more recent report showing reduced FA in cerebellar output pathways in ASD (Catani et al. 2008).

To our knowledge, this is the first report of the properties of FA distributions in young children with autism. The abnormal \&values, driven by the standard deviation of the transformed $F$ distributions, may reflect more homogeneous white matter in autism, typical of what would be expected in younger children. Although the use of a novel statistical method was a unique advantage, the study was limited by the large neuroanatomical regions from which the FA values were extracted. Current research in our group focuses on new methods for population-based statistics of individual fibre tracts (Goodlett et al. 2006), segmented from high-resolution DTI on three Tesla scanners.

The variability in image slice thickness was another limitation intrinsic to the imaging hardware and/or acquisition software. This variable did not affect any group differentially, thus our findings are not compromised, but this is a potential confounding variable that future studies should take steps to monitor.

An important finding was the patterns predicted trajectories of the three groups throughout the age range of the sample ( 2 to 6 years). In the lobes analysis, a small group of children with non-specific DD maintained a steady level of $\delta$ relative to controls throughout the age range. As can be seen from Fig. 3, this translates to an abnormally high level of $\delta_{\text {in }}$ DD relative to the typical group until about age 3 or 4 , at which point the typical control group, which starts with lower $\delta$ but increases steadily, catches up and surpasses the DD sample. It is important to note, however, that although the overall interaction between group and age was significant for the lobes analysis, it was not for the global analysis, and that contrasts among the three groups only showed significant interactions between group and age for the typical and DD groups. It is possible that increased heterogeneity in the ASD group limited statistical power to detect the difference from the DD group that is suggested by Figs 2-4. Our preliminary analyses separating the components of $\delta$ suggest that these differences 
reflect the predicted individual variability in FA among the groups. This suggests that an autismspecific reduction of variability in white matter diffusion, beyond what is seen in general delay, is present in the first 5 years of life. In addition, although the size of the DD group limits definitive conclusions, the flat slope in the DD group is consistent with a longitudinal study by Shaw et al. (2006) in which the trajectory of change in cortical thickness was associated with generalised intellectual ability, suggesting that early brain plasticity is closely related to cognitive development. This early window is a critical time in neural development, and the impact of early white matter organisation is fundamental to properly functioning neural networks that process information and allow behavioural adaptation throughout the lifespan. A better understanding of emerging neural connections during this window has potential to yield biomarkers or other important tools that could be used clinically in the assessment and treatment of autism.

Future studies should focus on: (1) more comprehensive exploration of the variability in FA distributions; (2) longitudinally following this and other samples to determine how the trajectories change in later childhood and adolescence; (3) combining DTI measures in more circumscribed areas, for example in well defined fibre tracts of interest associated with specific brain function, with functional imaging to elucidate the most important neural networks affected in autism; (4) replication with a larger idiopathic DD group; and (5) relating the dysfunction in these systems to the complement of behavioural symptoms that characterise autism.

\section{Supplementary Material}

Refer to Web version on PubMed Central for supplementary material.

\section{Acknowledgments}

This work was supported by NIH grants R01 MH61696, R01 MH64580 and P30 HD03110 (PI: J. Piven).

\section{References}

Adolphs R, Sears L, Piven J. Abnormal processing of social information from faces in autism. Journal of Cognitive Neuroscience. 2001; 11:231-9.

Alexander AL, Lee JE, Lazar M, Boudos R, DuBray MB, Oakes TR, et al. Diffusion tensor imaging of the corpus callosum in autism. NeuroImage. 2007; 34:61-73. [PubMed: 17023185]

Amaral DG, Corbett BA. The amygdala, autism, and anxiety. Novartis Foundation Symposium. 2003; 251:177-87. [PubMed: 14521193]

American Psychiatric Association. Diagnostic and Statistical Manual of Mental Disorders. 4. American Psychiatric Association; Arlington, VA: 2000. Text Revision

Aylward EH, Minshew NJ, Goldstein G, Honeycutt NA, Augustine AM, Yates KO, et al. MRI volumes of amygdala and hippocampus in non-mentally retarded autistic adolescents and adults. Neurology. 1999; 53:2145-50. [PubMed: 10599796]

Barnea-Goraly N, Kwon H, Menon V, Eliez S, Lotspeich L, Reiss AL. White matter structure in autism: preliminary evidence from diffusion tensor imaging. Biological Psychiatry. 2004; 55:323-6. [PubMed: 14744477]

Barnea-Goraly N, Lotspeich L, Reiss AL. Similar white matter aberrations in children with autism and their unaffected siblings: a diffusion tensor imaging study using tract-based spatial statistics. Archives of General Psychiatry. 2010; 67:1052-60. [PubMed: 20921121]

Basser PJ, Pierpaoli C. Microstructural and physiological features of tissues elucidated by quantitativediffusion-tensor MRI. Journal of Magnetic Resonance Series B. 1996; 111:209-19. [PubMed: 8661285]

Bauman M, Kemper TL. Histoanatomic observations of the brain in early infantile autism. Neurology. 1985; 25:866-74. [PubMed: 4000488] 
Beaulieu C. The basis of anisotropic water diffusion in the nervous system - a technical review. NMR in Biomedicine. 2002; 15:435-55. [PubMed: 12489094]

BenBashat D, Kronfeld-Duenias V, Zachor DA, Ekstein PM, Hendler T, Tarrasch R, et al. Accelerated maturation of white matter in young children with autism: a high b value DWI study. NeuroImage. 2007; 37:40-7. [PubMed: 17566764]

Boddaert N, Chabane N, Gervais H, Good CD, Bourgeois M, Plumet MH, et al. Superior temporal sulcus anatomical abnormalities in childhood autism: a voxel-based morphometry MRI study. NeuroImage. 2004; 23:364-9. [PubMed: 15325384]

Brown, H.; Prescott, R. Applied Mixed Models in Medicine. 2. Wiley; NewYork: 2006.

Carper RA, Courchesne E. Localized enlargement of the frontal cortex in early autism. Biological Psychiatry. 2005; 57:126-33. [PubMed: 15652870]

Cascio CJ, Gerig G, Piven J. Diffusion tensor imaging: application to the study of the developing brain. Journal of the American Academy of Child and Adolescent Psychiatry. 2007; 46:213-23. [PubMed: 17242625]

Catani M, Jones DK, Daly E, Embiricos N, Deeley Q, Pugliese L, et al. Altered cerebellar feedback projections in Asperger syndrome. NeuroImage. 2008; 41:1184-91. [PubMed: 18495494]

Clement, M.; Couper, D.; Marron, S.; Muller, KE.; Zhu, H. Analyzing diffusion tensor imaging data. Presented at the Annual Joint Statistical Meeting; Denver, CO. 2008.

Clement-Spychala ME, Couper D, Zhu H, Muller KE. Approximating the Geisser-Greenhouse sphericity estimator and its applications to diffusion tensor imaging. Statistics and Its Interface. 2010; 3:81-90.

Courchesne E, Pierce K. Why the frontal cortex in autism might be talking only to itself: local overconnectivity but long-distance disconnection. Current Opinion in Neurobiology. 2005; 15:225-30. [PubMed: 15831407]

Courchesne E, Karns CM, Davis HR, Ziccardi R, Carper RA, Tigue ZD, et al. Unusual brain growth patterns in early life in patients with autistic disorder: an MRI study. Neurology. 2001; 57:245-54. [PubMed: 11468308]

Frith C. What do imaging studies tell us about the neural basis of autism? Novartis Foundation Symposium. 2003; 251:149-66. [PubMed: 14521192]

Geschwind DH, Levitt P. Autism spectrum disorders: developmental disconnection syndromes. Current Opinion in Neurobiology. 2007; 17:103-11. [PubMed: 17275283]

Goodlett C, Davis B, Jean R, Gilmore J, Gerig G. Improved correspondence for DTI population studies via unbiased atlas building. Medical Image Computing and Computer-Assisted Intervention. 2006; 9:260-7. [PubMed: 17354780]

Hazlett HC, Poe MD, Gerig G, Smith RG, Provenzale J, Ross A, et al. Magnetic resonance imaging and head circumference study of brain size in autism. Archives of General Psychiatry. 2005; 62:1366-76. [PubMed: 16330725]

Jou RJ, Mateljevic N, Kaiser MD, Sugrue DR, Volkmar FR, Pelphrey KA. Structural neural phenotype of autism: preliminary evidence from a diffusion tensor imaging study using tract-based spatial statistics. AJNR American Journal of Neuroradiology. 2011; 32:1607-13. [PubMed: 21799040]

Keller TA, Kana RK, Just MA. A developmental study of the structural integrity of white matter in autism. Neuroreport. 2007; 18:23-7. [PubMed: 17259855]

Kumar A, Sundaram SK, Sivaswamy L, Behen ME, Makki MI, Ager J, et al. Alterations in frontal lobe tracts and corpus callosum in young children with autism spectrum disorder. Cerebral Cortex. 2010; 20:2103-13. [PubMed: 20019145]

Langen M, Leemans A, Johnston P, Ecker C, Daly E, Murphy CM, et al. Fronto-striatal circuitry and inhibitory control in autism: findings from diffusion tensor imaging tractography. Cortex. 2011; 48:183-93. [PubMed: 21718979]

LeCouteur, A.; Lord, C.; Rutter, M. The Autism Diagnostic Interview - Revised (ADI-R). Western Psychological Corporation; Los Angeles: 2003.

Lee JE, Bigler ED, Alexander AL, Lazar M, DuBray MB, Chung MK, et al. Diffusion tensor imaging of white matter in the superior temporal gyrus and temporal stem in autism. Neuroscience Letters. 2007; 424:127-32. [PubMed: 17714869] 
Lo YC, Soong WT, Gau SS, Wu YY, Lai MC, Yeh FC, et al. The loss of asymmetry and reduced interhemispheric connectivity in adolescents with autism: a study using diffusion spectrum imaging tractography. Psychiatry Research. 2011; 192:60-6. [PubMed: 21377337]

Lord, C.; Rutter, M.; DiLavore, P.; Risi, S. The Autism Diagnostic Observation Schedule (ADOS). Western Psychological Corporation; Los Angeles: 1999.

McAlonan GM, Cheung V, Cheung C, Suckling J, Lam GY, Tai KS, et al. Mapping the brain in autism. A voxel-based MRI study of volumetric differences and intercorrelations in autism. Brain. 2005; 128:268-76. [PubMed: 15548557]

Mengotti P, D’Agostini S, Terlevic R, De Colle C, Biasizzo E, Londero D, et al. Altered white matter integrity and development in children with autism: a combined voxel-based morphometry and diffusion imaging study. Brain Research Bulletin. 2011; 84:189-95. [PubMed: 21146593]

Minshew NJ, Keller TA. The nature of brain dysfunction in autism: functional brain imaging studies. Current Opinion in Neurology. 2010; 23:124-30. [PubMed: 20154614]

Minshew NJ, Williams DL. The new neurobiology of autism: cortex, connectivity, and neuronal organization. Archives of Neurology. 2007; 64:945-50. [PubMed: 17620483]

Mosconi MW, Mack PB, McCarthy G, Pelphrey KA. Taking an 'intentional stance' on eye-gaze shifts: a functional neuroimaging study of social perception in children. NeuroImage. 2005; 27:247-52. [PubMed: 16023041]

Muller, KE.; Fetterman, BA. Regression and ANOVA: An Integrated Approach Using SAS ${ }^{\circledR}$ Software. SAS Publishing; Cary, NC: 2002.

Müller RA. The study of autism as a distributed disorder. Mental Retardation and Developmental Disabilities Research Reviews. 2007; 13:85-95. [PubMed: 17326118]

Noonan SK, Haist F, Müller RA. Aberrant functional connectivity in autism: evidence from lowfrequency BOLD signal fluctuations. Brain Research. 2009; 1262:48-63. [PubMed: 19401185]

Palmen SJ, Hulshoff HE, Kemner C, Schnack HG, Durston S, Lahuis BE, et al. Increased gray-matter volume in medication-naïve high-functioning children with autism spectrum disorder. Psychological Medicine. 2005; 35:561-70. [PubMed: 15856726]

Piven J, Arndt S, Bailey J, Andreasen N. Regional brain enlargement in autism: a magnetic resonance imaging study. Journal of the American Academy of Child and Adolescent Psychiatry. 1996; 35:530-6. [PubMed: 8919716]

Puff DT, Pisano ED, Muller KE, Johnston RE, Hemminger BM, Burbeck CA, et al. A method for determination of optimal image enhancement for the detection of mammographic abnormalities. Journal of Digital Imaging. 1994; 7:161-71. [PubMed: 7858011]

Pugliese L, Catani M, Ameis S, Dell'Acqua F, Thiebaut de Schotten M, Murphy C, et al. The anatomy of extended limbic pathways in Asperger syndrome: A preliminary diffusion tensor imaging tractography study. NeuroImage. 2009; 47:427-34. [PubMed: 19446642]

Schmitz N, Rubia K, Daly E, Smith A, Williams S, Murphy DG. Neural correlates of executive function in autism spectrum disorders. Biological Psychiatry. 2006; 59:7-16. [PubMed: 16140278]

Schultz RT, Gauthier I, Klin A, Fulbright RK, Anderson AW, Volkmar F, et al. Abnormal ventral temporal cortical activity during face discrimination among individuals with autism and Asperger syndrome. Archives of General Psychiatry. 2000; 57:331-40. [PubMed: 10768694]

Sears LL, Vest C, Mohamed S, Bailey J, Ranson BJ, Piven J. An MRI study of the basal ganglia in autism. Progress in Neuro-Psychopharmacology \& Biological Psychiatry. 1999; 23:613-24. [PubMed: 10390720]

Shafritz KM, Dichter GS, Baranek GT, Belger A. The neural circuitry mediating shifts in behavioral response and cognitive set in autism. Biological Psychiatry. 2008; 63:974-80. [PubMed: 17916328]

Shaw P, Greenstein D, Lerch J, Clasen L, Lenroot R, Gogtay N, et al. Intellectual ability and cortical development in children and adolescents. Nature. 2006; 440:676-9. [PubMed: 16572172]

Shukla DK, Keehn B, Müller RA. Tract-specific analyses of diffusion tensor imaging show widespread white matter compromise in autism spectrum disorder. Journal of Child Psychology and Psychiatry. 2011; 52:286-95. [PubMed: 21073464] 
Sparks BF, Friedmand SD, Shaw DW, Aylward EH, Echelard D, Artru AA, et al. Brain structural abnormalities in young children with autism spectrum disorder. Neurology. 2002; 59:184-92. [PubMed: 12136055]

Turner MA. Generating novel ideas: fluency performance in high-functioning and learning disabled individuals with autism. Journal of Child Psychology and Psychiatry. 1999; 40:189-201. [PubMed: 10188701]

Weinstein M, Ben-Sira L, Levy Y, Zachor DA, Ben Itzhak E, Artzi M, et al. Abnormal white matter integrity in young children with autism. Human Brain Mapping. 2011; 32:534-43. [PubMed: 21391246]

Yushkevich P, Piven J, Cody Hazlett H, Gimpel Smith S, Gee J, Gerig G. User-guided 3D active contour segmentation of anatomical structures: significantly improved efficiency and reliability. NeuroImage. 2006; 31:1116-28. [PubMed: 16545965] 

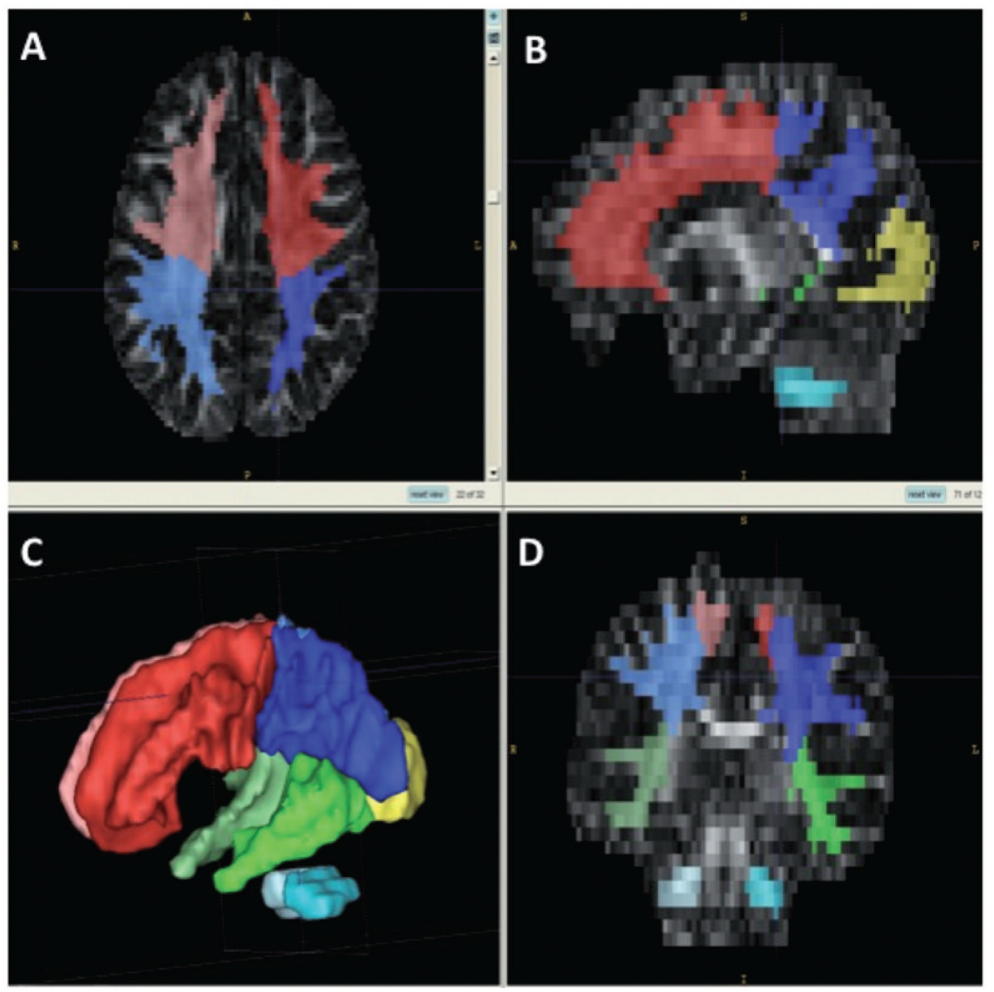

Figure 1.

Creation of cortical regions of interest. Lower left panel (C): cortical lobe parcellation map. Panels A, B and D: axial, sagittal and coronal views of the thresholded white matter mask superimposed on the cortical segmentation map. 


\section{Global White Matter: Scatter Plot with Age Trajectories}

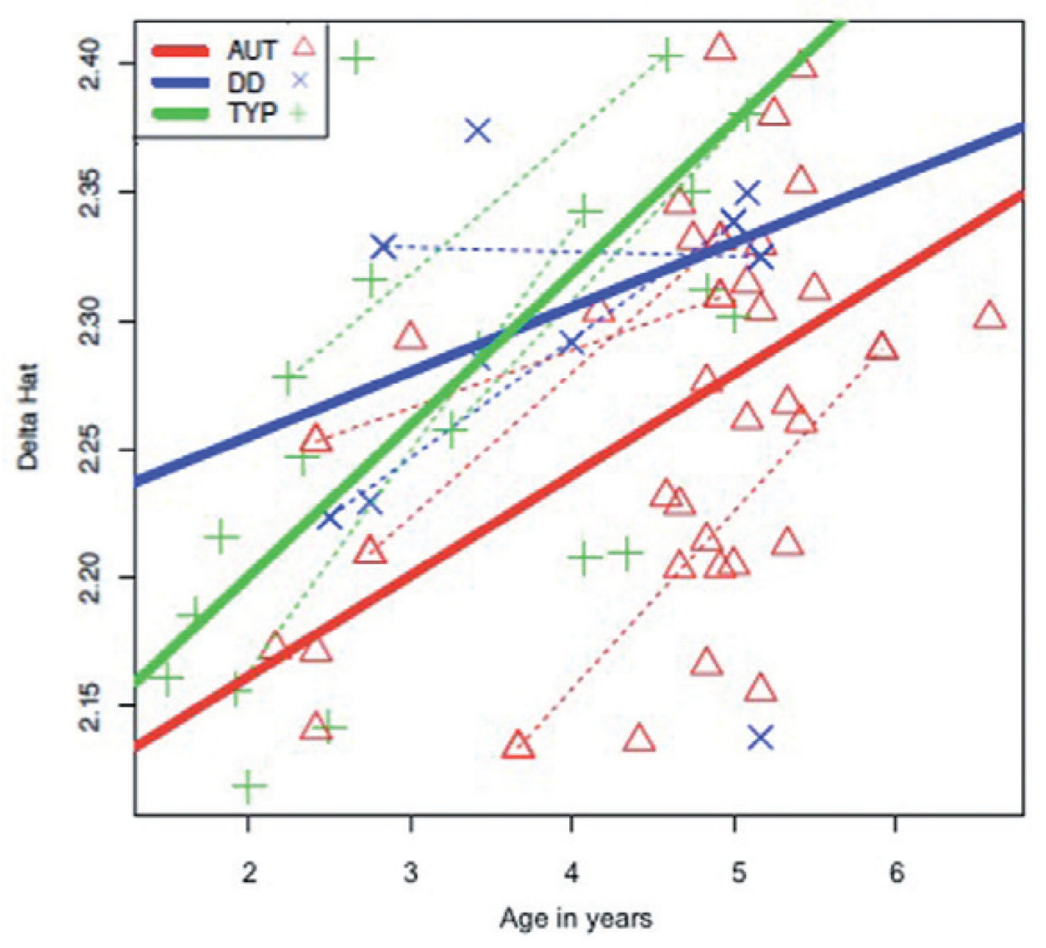

Figure 2.

Predicted global $\delta$ estimated using Restricted Maximum Likelihood Estimation mixed model as a function of age by group. Dotted line: subjects with repeated measures. AUT, autism; DD, developmental delay; TYP, typically developing. 
Lobes White Matter: Scatter Plot with Age Trajectories

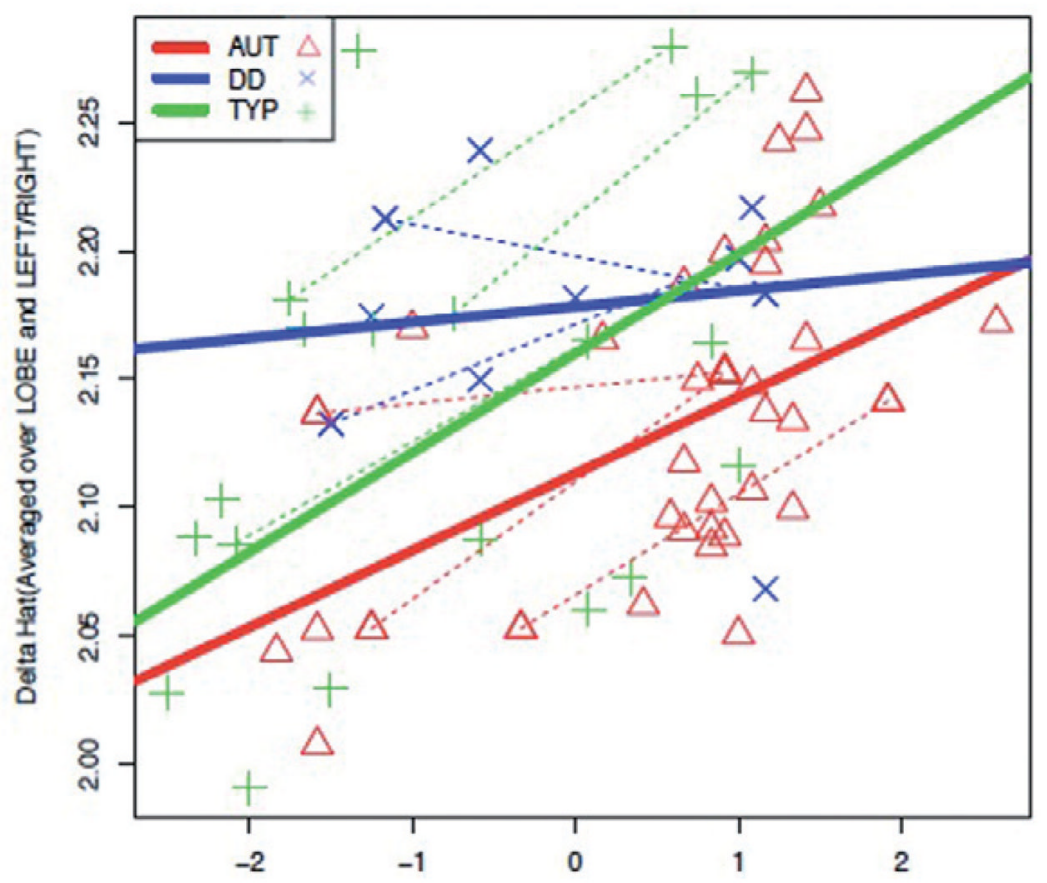

Figure 3.

Predicted $\delta$ estimated using Restricted Maximum Likelihood Estimation mixed model averaged across lobes and hemispheres as a function of age by group. Dotted line: subjects with repeated measures. AUT, autism; DD, developmental delay; TYP, typically developing. 


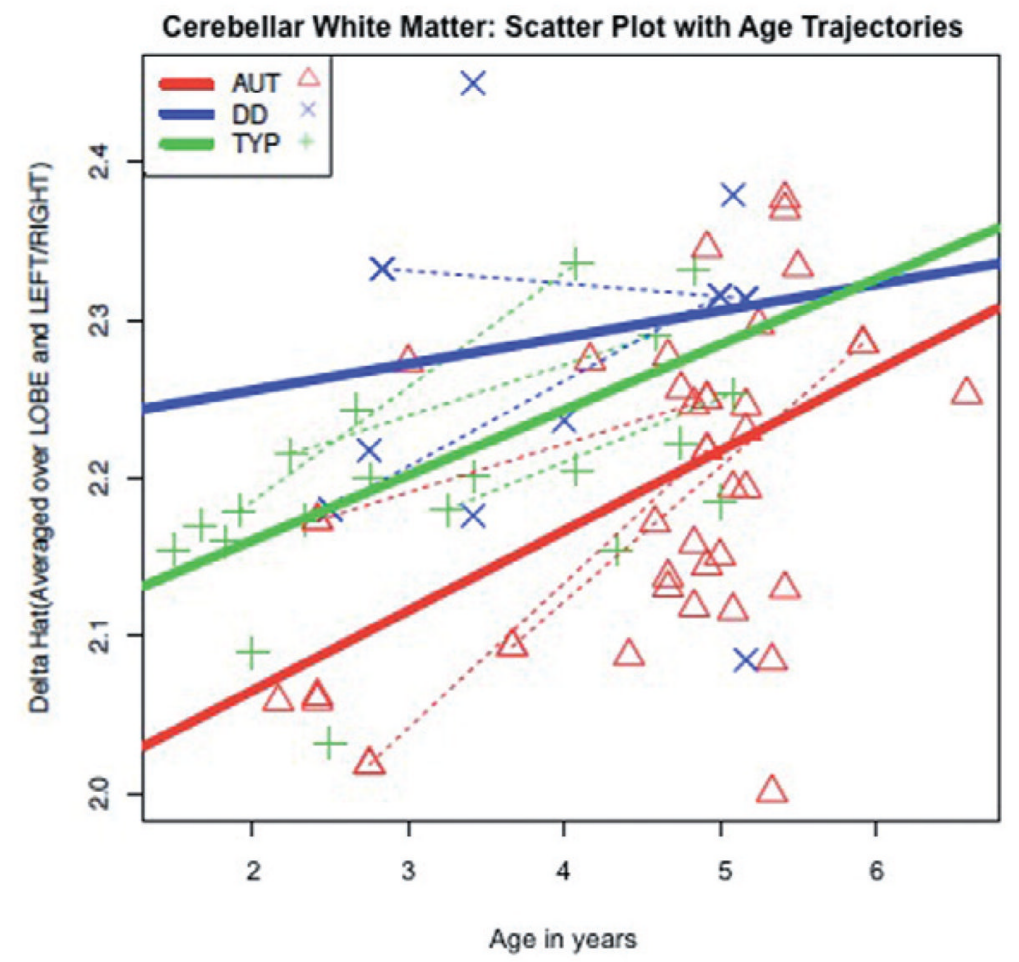

Figure 4.

Predicted cerebellar $\delta$ estimated using Restricted Maximum Likelihood Estimation mixed model as a function of age by group. Dotted line: subjects with repeated measures. AUT, autism; DD, developmental delay; TYP, typically developing. 


\section{Table 1}

Summary demographic data on the sample

\begin{tabular}{lcccc}
\hline & Autism & TYP & DD & Total \\
\hline N participants & 33 & 17 & 8 & 58 \\
N observations & 36 & 20 & 10 & 66 \\
IQ * [Mean (SD)] & $57.3(17.3)$ & $110.5(18)$ & $58.9(10.1)$ & $73.1(29.3)$ \\
VIQ & $53.9(12.9)$ & $95.9(10.4)$ & $64.2(13.7)$ & \\
PIQ & $25.6(10.1)$ & $54.9(8.6)$ & $28.7(8.2)$ & \\
Age [Mean years (SD)] & $4.6(1.1)$ & $3.2(1.3)$ & $3.93(1.1)$ & $4.1(1.3)$ \\
$\%$ Male & 91.7 & 80 & 50 & 81.8 \\
ADI Social Composite & $20.11(5.9)$ & - & - & \\
ADOS Communication + Social Interaction & $17.2(3.3)$ & - & - & \\
\hline
\end{tabular}

Age is the mean for all observations in the group.

* Estimated IQ, based on measures of cognitive development (Vineland Adaptive Behavior Scale), from which the communication domain was used to compute verbal IQ (VIQ) and Mullen Scales of Early Learning, from which the visual reception and fine motor subtests were used to compute performance IQ (PIQ).

TYP, typically developing; DD, developmentally delayed; ADI, Autism Diagnostic Interview (Social composite cut-off for autism - 10); ADOS, Autism Diagnostic Observation Schedule. 